\title{
Identifying New COVID-19 Receptor Neuropilin-1 in Severe Alzheimer's Disease Patients Group Brain Using Genome-Wide Association Study Approach
}

\author{
Key-Hwan Lim', Sumin Yang', Sung-Hyun Kim and Jae-Yeol Joo* \\ Neurodegenerative Disease Research Group, Korea Brain Research Institute, Daegu, South Korea
}

OPEN ACCESS

Edited by:

Noriyoshi Usui,

Osaka University, Japan

Reviewed by:

Kazuya Toriumi,

Tokyo Metropolitan Institute

of Medical Science, Japan

Sangwon Byun,

Korea Research Institute of Bioscience and Biotechnology

(KRIBB), South Korea

*Correspondence:

Jae-Yeol Joo

joojy@kbri.re.kr

${ }^{\dagger}$ These authors have contributed equally to this work

Specialty section:

This article was submitted to

Neurogenomics,

a section of the journal

Frontiers in Genetics

Received: 14 July 2021 Accepted: 02 August 2021 Published: 21 October 2021

Citation:

Lim K-H, Yang S, Kim S-H and Joo J-Y (2021) Identifying New COVID-19 Receptor Neuropilin-1

in Severe Alzheimer's Disease

Patients Group Brain Using

Genome-Wide Association Study

Approach. Front. Genet. 12:741175.

doi: 10.3389/fgene.2021.741175
Recent preclinical studies show that Neuropilin-1 (NRP1), which is a transmembrane protein with roles in neuronal development, axonal outgrowth, and angiogenesis, also plays a role in the infectivity of severe acute respiratory syndrome coronavirus 2 (SARSCoV-2). Thus, we hypothesize that NRP1 may be upregulated in Alzheimer's disease (AD) patients and that a correlation between AD and SARS-CoV-2 NRP1-mediated infectivity may exist as angiotensin converting enzyme 2 (ACE2). We used an AD mouse model that mimics $A D$ and performed high-throughput total RNA-seq with brain tissue and whole blood. For quantification of NRP1 in AD, brain tissues and blood were subjected to Western blotting and real-time quantitative PCR (RT-qPCR) analysis. In silico analysis for NRP1 expression in AD patients has been performed on human hippocampus data sets. Many cases of severe symptoms of COVID-19 are concentrated in an elderly group with complications such as diabetes, degenerative disease, and brain disorders. Total RNA-seq analysis showed that the Nrp1 gene was commonly overexpressed in the AD model. Similar to ACE2, the NRP1 protein is also strongly expressed in AD brain tissues. Interestingly, in silico analysis revealed that the level of expression for NRP1 was distinct at age and AD progression. Given that NRP1 is highly expressed in AD, it is important to understand and predict that NRP1 may be a risk factor for SARS-CoV-2 infection in AD patients. This supports the development of potential therapeutic drugs to reduce SARS-CoV-2 transmission.

Keywords: SARS-CoV-2, Neuropilin-1, Alzheimer's disease, genome-wide association study (GWAS), gene expression

\section{INTRODUCTION}

Severe acute respiratory syndrome coronavirus 2 (SARS-CoV-2) is being evaluated as a thirdhigh-risk contagious infection (Hu et al., 2020). People are still highly vulnerable to the ongoing and life-threatening COVID-19 pandemic, as FDA-authorized vaccines or beneficial treatments remain unavailable (Singh et al., 2020). The risk of severe complications that are eventually 
associated with high mortality is indicated in older people (Carstensen et al., 2020). Moreover, a bidirectional interrelation between neurological complications and COVID-19 is extensively reported (Verkhratsky et al., 2020).

Age-dependent vulnerability to SARS-CoV-2 has been associated with concomitant symptomatic infections (Liu et al., 2020; Wu et al., 2020). Alzheimer's disease (AD) is a highly destructive neurodegenerative disorder that mostly affects the elderly and is characterized by a progressive cognitive decline (Masters et al., 2015). Although various hypotheses have been proposed to explain its multifactorial properties (Liu et al., 2019), the exact mechanism and related features of $\mathrm{AD}$ remain obscure. An analysis of 627 patients suggests that $\mathrm{AD}$ is a risk factor for SARS-CoV-2 infection (Bianchetti et al., 2020).

Angiotensin converting enzyme 2 (ACE2) is required for SARS-CoV-2 infection. Recently, it is reported that the Ace 2 gene and protein expression are elevated in $\mathrm{AD}$ patients compared with in normal elderly individuals (Ding et al., 2020; Lim et al., 2020; Rahman et al., 2020). Consistent with these results, an increase in ACE2 expression results in an increased susceptibility to SARS-CoV-2 infection in elderly patients with AD. Furthermore, a recent study suggests that the transmembrane protein Neuropilin-1 (NRP1) also plays a role in SARS-CoV-2 infection (Daly et al., 2020; Mayi et al., 2021). Biochemical experiments and X-ray crystallography show that NRP1 strongly interacts with a polybasic sequence on the spike protein of SARS-CoV-2, which fits the C-end rule region (CendR) required for NRP1-peptide interaction (Daly et al., 2020; Song et al., 2020). NRP1 depletion with RNAi targeting Nrp1 mRNA inhibits the binding of the SARS-CoV-2 spike protein to NRP1 and, consequently, decreases the rate of viral infection (Daly et al., 2020; Song et al., 2020). In addition, a monoclonal antibody against the b1 1 2 domain of NRP1 reduces the infectivity of SARS-CoV-2 lentiviral pseudo-particles (Cantuti-Castelvetri et al., 2020). NRP1 is a neuronal receptor associated with the regulation of neurite outgrowth through the binding of vascular endothelial growth factor (VEGF) (Abdullah et al., 2020). When NRP1 is activated by CendR, which is a peptide $\mathrm{R} / \mathrm{KXXR} / \mathrm{K}$ motif contained within C-terminal domains, it enables cells to internalize ligands, such as viruses, containing the motif (Teesalu et al., 2009). Furthermore, NRP1 is expressed in the central nervous system, including the brain olfactory-related regions in which SARS-CoV-2 entry may occur, thereby facilitating COVID-19 infection (Davies et al., 2020).

Thus, we hypothesize that, in addition to ACE2, NRP1 expression might be upregulated in the brains of elderly $\mathrm{AD}$ patients. In this study, molecular characterization via highthroughput analysis and biochemical assays reveals that NRP1 is highly expressed in AD, which suggests that NRP1 may be a potential genetic therapy target in AD patients with COVID-19.

\section{MATERIALS AND METHODS}

\section{Animals}

Five $\times$ FAD transgenic mice were purchased from the Jackson Laboratory. All animal experiments performed in this study were reviewed and approved by the IACUC committee at the Korea Brain Research Institute (IACUC-20-00018).

\section{Total RNA Sequencing and Human in silico Analysis}

The data analysis of total RNA-seq from the mouse cortex was performed as previously described in Lim and Joo (2020). Briefly, the brain was extracted from 6-month-old wild-type (WT) and $5 \times$ FAD mice and cortex isolated to prepare the pure RNA and total RNA-seq library. RNA-seq libraries were prepared using the TruSeq Stranded Total RNA LT Sample Prep Kit (Illumina Sample Preparation Guide) from isolated mRNA. To profile the insert length of libraries, we used the Agilent 2100 Bioanalyzer, and constructed libraries were sequenced from $\mathrm{HiSeq}^{\mathrm{TM}} 4000$ platform (Illumina, United States). Then, converted nucleotide sequences using $\mathrm{HiSeq}^{\mathrm{TM}} 4000$ were sorted and the dirty reads filtered from the raw reads. RNA-seq data was accessible using Gene Expression Omnibus (GEO) accession number GSE147792. In silico data analysis was performed using the Affymetrix Human Genome U133 Plus 2.0 Array (Lim et al., 2020). The GSE1297 data sets were derived from human hippocampus and GSE4226 data sets were derived from human peripheral blood mononuclear cells (PBMCs) in normal and AD patients.

\section{RNA Isolation}

Total RNA isolation was performed with the mouse cortex according to TRIzol using the commercial protocol. First, phenolbased TRIzol (Invitrogen) is added in the cortex tissue tube for homogenizing. Then, it is separated into three phases by chloroform for the collect only RNA dissolved aqueous phase except the DNA and protein precipitated phases. An equal volume of isopropanol was used to precipitate RNA. After centrifugation, supernatant was discarded, and it was washed with prechilled $75 \%$ ethanol once. RNA was dehydrated and crystalized without organic compound contamination and eluted with nuclease-free water. RNA was then denatured in the $65^{\circ} \mathrm{C}$ heat block for $10 \mathrm{~min}$. The procedure was performed without RNase contamination.

\section{Complementary DNA Synthesis}

Isolated total RNA was synthesized into complementary DNA (cDNA) following the manufacturer's protocol of High-Capacity cDNA Reverse Transcription Kits (Applied Biosystems). Template RNA (2 $\mu \mathrm{g}$ ) was prepared to synthesize a single reaction, and reverse transcription kit components were premixed. The premixture contains $10 \times$ RT buffer, $25 \times \mathrm{dNTP}$ mix $(4 \mathrm{mM}), 10 \times$ RT Random Primers, MultiScribe Reverse Transcriptase (50 U), RNase inhibitor, and nuclease-free water for adjusting the total volume for the reaction. Gently mixed template RNA and an equal volume of premixture was placed in the thermal cycler. The condition for reverse transcription was suggested as optimized temperature and time: $25^{\circ} \mathrm{C}$ for $10 \mathrm{~min}$, $37^{\circ} \mathrm{C}$ for $120 \mathrm{~min}$, and $85^{\circ} \mathrm{C}$ for $5 \mathrm{~min}$. 


\section{Real-Time Quantitative PCR}

Real-time quantitative PCR (RT-qPCR) was performed according to commercial protocol using SYBR Green PCR Master Mix (Applied Biosystems). Primers employed were $N r p 1$ forward, 5' CCTCACATTGGGCGTTATTG 3', reverse, 5' CACTGTAGTTGGCTGAGAAAC $3^{\prime}$; Gapdh forward, $5^{\prime}$ AGGTCGGTGTGAACGGATTT $3^{\prime}$, reverse, $5^{\prime}$
TGTAGACCATGTAGTTGAGG $3^{\prime}$. Each reaction contains SYBR Green PCR Master Mix, Template cDNA, and forward and reverse primer and is adjusted with nuclease-free water.

\section{Western Blot}

Protein was extracted from the mouse cortex and mixed with sample buffer (5\% 2-mercaptoethanol) and boiled at $100^{\circ} \mathrm{C}$ for

A

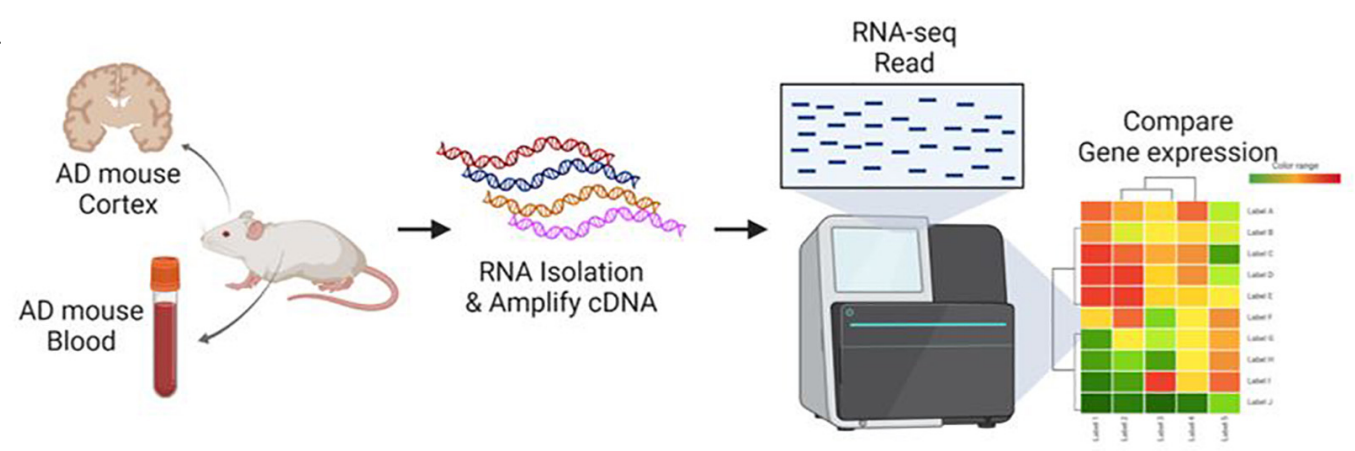

B
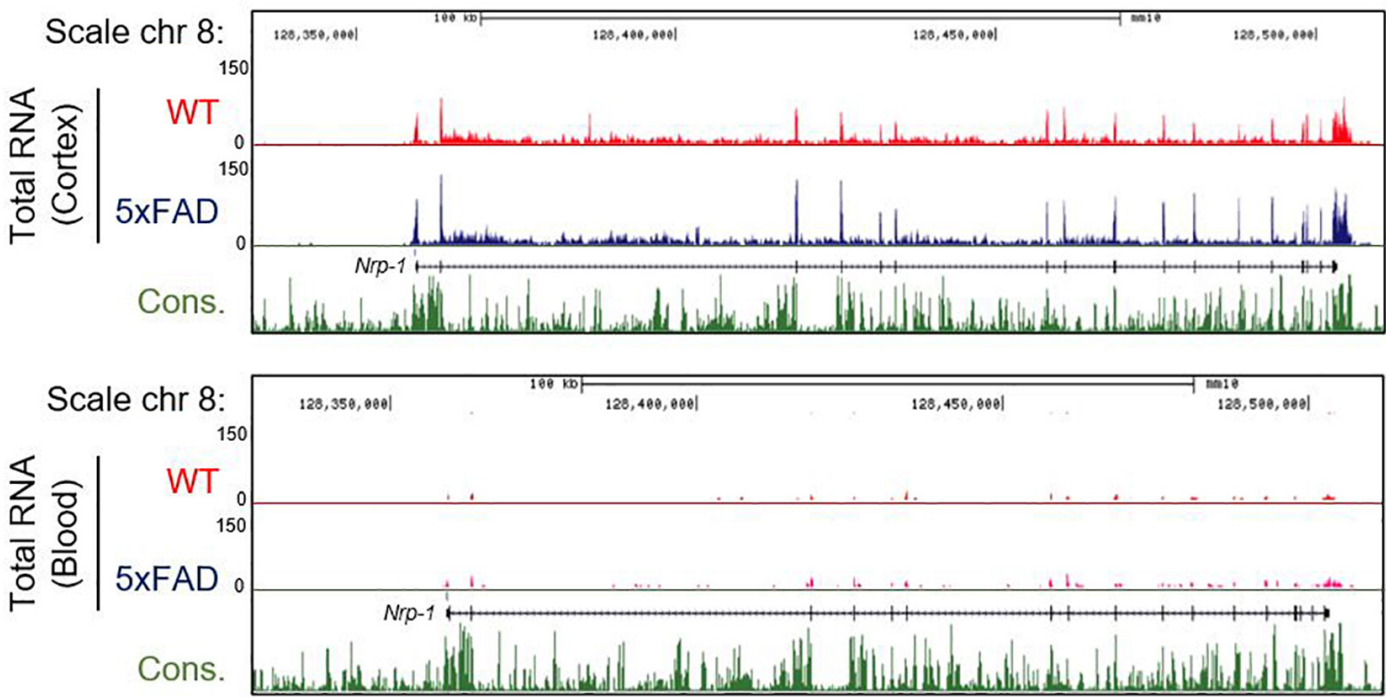

C

Neuropilin-1

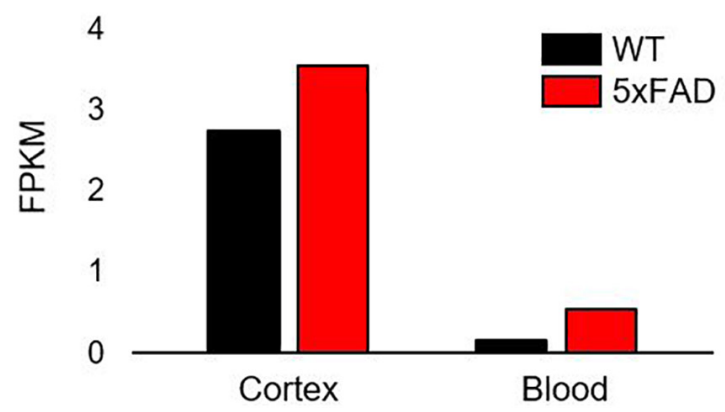

FIGURE 1 | Correlation between Nrp1 gene and protein expression in AD brain. (A) Graphic representation of Nrp1 gene expression in the cortex of five familial AD mutations (5 $\times$ FAD) mice, which are used as a murine model of AD. (B) The representation is shown on the UCSC genome browser following total RNA-seq. (C) Nrp1 gene expression levels are increased by $129 \%$ in 5xFAD cortex compared with control WT cortex. 
denaturation. Protein samples were loaded on $4-15 \%$ gradient gel (Bio-Rad) to separate by size through the vertical SDSPAGE system. Antibodies used for immune-blot analysis were anti-Neuropilin-1 (abcam, 1:1000) and anti- $\beta$-actin (BETHYL, 1:10000). Images were acquired by ChemiDoc MP imaging system (Bio-Rad).

\section{RESULTS}

\section{High-Throughput Analysis of Nrp1 Expression in Alzheimer's Disease}

Given that the gene expression of ACE2 is upregulated in the brains of patients with $\mathrm{AD}$ and may be associated with the mortality rate from COVID-19 in the elderly (Fu et al., 2020; Lim et al., 2020), we hypothesize that NRP1, which codes for a newly recognized SARS-CoV-2 spike receptor, may be also increased in $\mathrm{AD}$ patients. To assess $\mathrm{Nrp} 1$ gene expression in $\mathrm{AD}$, we first used a murine model that mimics $\mathrm{AD}$ and performed total RNA-seq using mouse brain tissue and whole blood. Total RNA-seq was analyzed by the HiSeq ${ }^{\mathrm{TM}} 4000$ platform (Illumina, United States) (Figure 1A). We applied the Nrp1 gene expression level in the brain and blood from $\mathrm{AD}$ and WT and then mapped the sequencing reads (Figure 1B). The track of Nrp1 gene was displayed with University of California, Santa Cruz (USCS) genome browser (Figure 1B). Interestingly, total RNAseq analysis revealed upregulation of $\mathrm{Nrp} 1$ gene expression in the brain of the AD model compared to WT (Figure 1B), and Nrp1 fragments per kb per million reads values are increased in the AD model brain as well (Figure 1C). Although Nrp1 gene expression was increased by $319 \%$ in $\mathrm{AD}$ blood compared with WT blood, the endogenous expression levels of Nrp1 in the blood were significantly lower than those in the brain (Figures 1B,C). Collectively, our total RNA-seq results show that
A

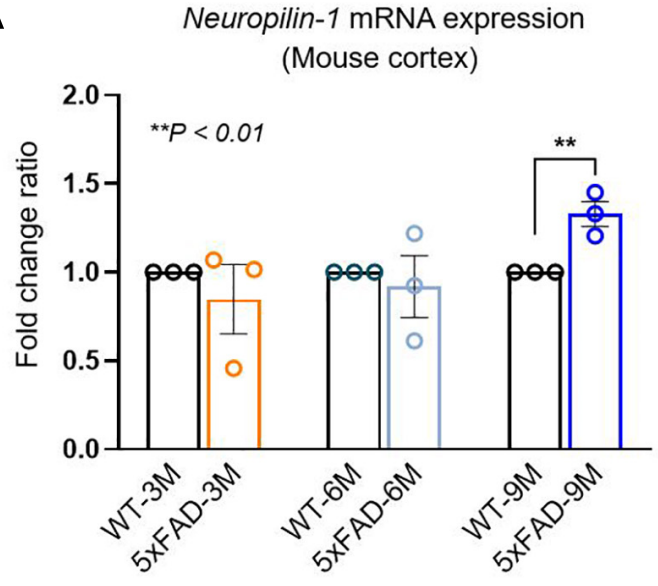

B

Mouse cortex (9-month)

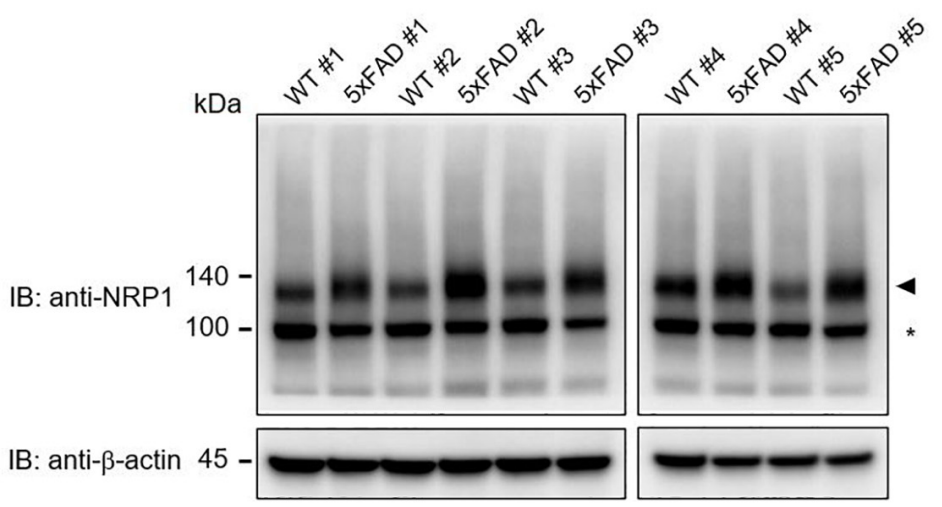

C
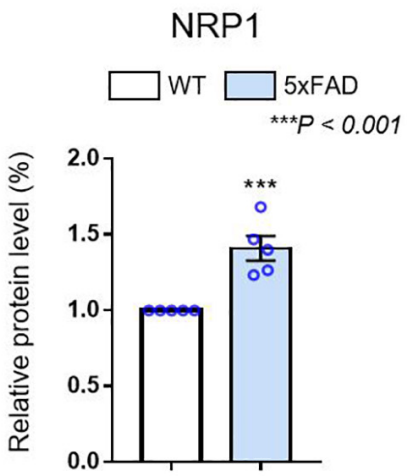

FIGURE 2 | The expression of NRP1 in mouse AD brain. (A) RT-qPCR analysis showing the Nrp1 mRNA expression levels in the cortex of WT and $5 \times$ FAD mice. Nrp1 mRNA expression is significantly increased in 9-month-old 5xFAD cortex compared with that in WT cortex. No significant differences are observed in the early disease stages of $5 \times$ FAD mice ( 3 and 6 months). The data are shown as the mean \pm standard error of the mean (SEM) from $n=3$ mice per group; statistical differences were assessed using unpaired $t$-test. (B) Representative Western blot analyzing the NRP1 protein levels in $5 \times$ FAD brains. Endogenous NRP1 is highly expressed in 9-month-old 5xFAD brains compared with that in the WT brain. $\beta$-actin was used as a loading control. The arrowhead indicates the NRP1 protein, and the asterisk indicates a non-specific band ( $n=5$ mice per group). (C) NRP1 Western blot band intensity measured by Image 1.50 i software ( $n=5$ mice per group). Statistical differences were assessed using unpaired $t$-test. 
Nrp1 is preferentially expressed in the brain and upregulated in the brains of AD mice.

\section{Nrp1 Is Upregulated in Alzheimer's Disease Brain}

Nrp1 is abundantly expressed in the neurons and plays an important role for axon guidance, regeneration, neuronal plasticity, or various human diseases, such as epilepsy and seizure (Kumanogoh and Kikutani, 2013).

We confirmed Nrp1 gene expression in both WT and AD model mouse brains through the total RNA-seq (Figure 1). To further analyze Nrp1 expression during AD progression, we measured the Nrp1 mRNA levels in 3- to 9-month-old AD brains. RT-qPCR revealed an approximately $145 \%$ increase in Nrp1
mRNA expression in 9-month-old AD brains compared with that in WT brains (Figure 2A). In addition, NRP1 protein expression was also significantly increased in 9-month-old $\mathrm{AD}$ brains compared with that in the WT (Figures 2B,C). Taken together, these findings indicate that NRP1 gene and protein expression levels are significantly increased in the brains of aged $\mathrm{AD}$ mice.

\section{Severe Alzheimer's Disease Patients Are Highly Expressed With Nrp1}

Having found increased Nrp1 gene expression in the brains of AD mice, we next performed Nrp1 gene expression profiling of brains and PBMCs from human patients with different stages of $\mathrm{AD}$ (Supplementary Table 1). To identify the fold change of the ratio for Nrp1 gene from AD patients, we performed in silico analysis

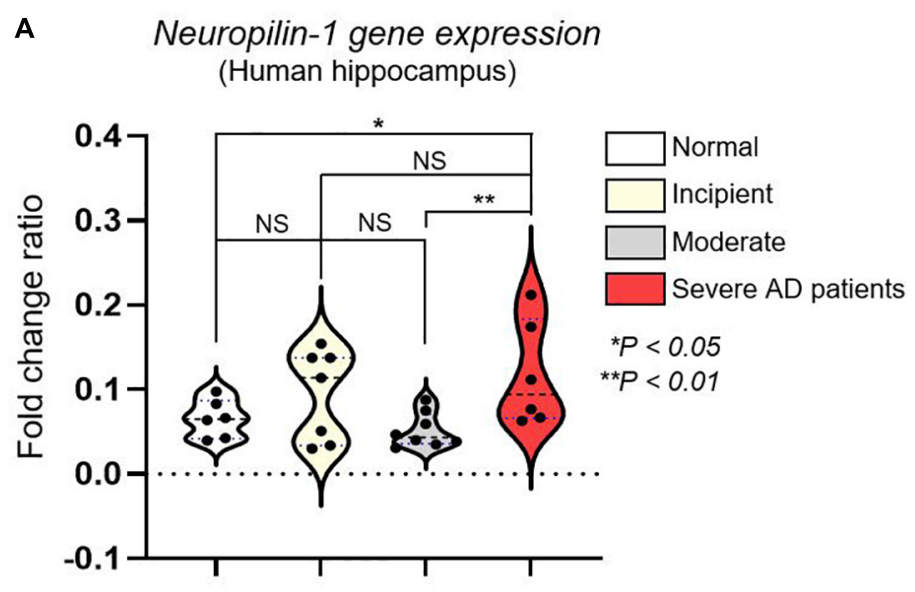

B

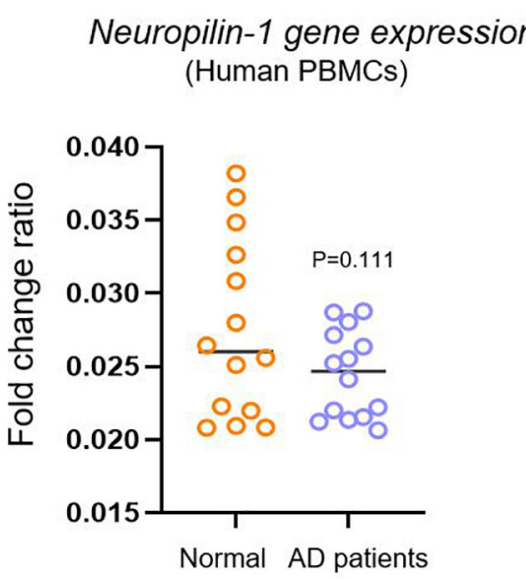

C

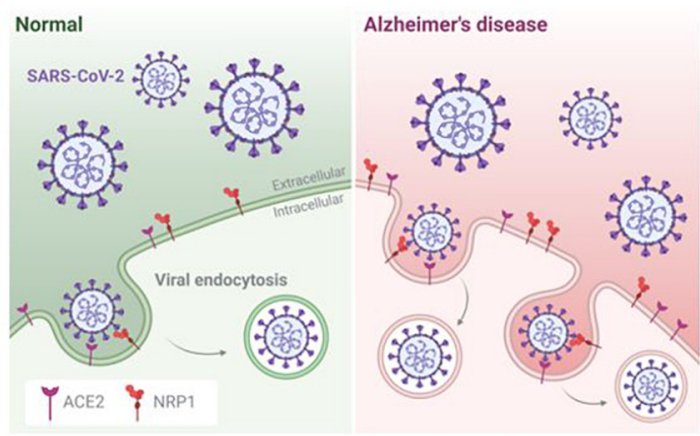

FIGURE 3 | In silico analysis of Nrp1 gene expression in human hippocampus and PBMCs from AD patients. (A) Nrp1 expression is significantly increased in the human hippocampus of severe AD patients compared with that in the control group (179\%). No statistical difference is observed when WT is compared to incipient and moderate AD patients. Normal control group $n=6$, incipient group $n=7$, moderate group $n=8$, and severe group $n=6$. Statistical differences were assessed using post hoc test after one-way ANOVA. (B) Nrp1 expression in PBMCs from AD patients is not statistically different from that in the control group. Normal elderly control, female $n=7$ and male $n=7$; AD patient group, female $n=7$ and male $n=7$. Statistical differences were assessed using unpaired $t$-test. (C) Schematic model of NRP1- and ACE2-mediated SARS-CoV-2 infection in AD. NRP1 and ACE2 mediate SARS-CoV-2 binding to the cell membrane and, consequently, infection. Because these two receptors are highly expressed in AD patients, these individuals may be more sensitive to SARS-CoV-2 infection. 
using the GSE1297 and GSE4296 microarray data set. Patients with severe AD showed significantly upregulated Nrp1 gene expression (179\%) compared with the control group (individuals without $\mathrm{AD}$ ), whereas incipient and moderate $\mathrm{AD}$ patients did not show increases in brain $N r p 1$ gene expression (Figure 3A). Interestingly, we did not find differences in PBMC Nrp1 gene expression between any of the groups (Figure 3B). These data correlate with results from the AD murine model. Together, the results demonstrate that NRP1 mRNA and protein expression is significantly elevated in the brains of late-stage $\mathrm{AD}$ patients.

\section{DISCUSSION}

Since the beginning of the COVID-19 pandemic, there have been significant efforts to identify unique SARS-CoV-2-associated proteins that could serve as targets for novel vaccines or therapeutic agents. Despite notable studies suggesting the possibility of developing other COVID-19-targeted drugs, the first-generation drugs have mostly focused on the viral spike protein receptor ACE2 (Yin et al., 2020). As high-throughput genomic studies begin to define the abnormal expression of individual DNA in particular diseases, it may become possible to rationally determine disease-specific gene expression and, thus, establish biomarkers for risk prediction in older people with complications, such as AD. Recently, we showed the increase of ACE2 expression in an elderly group with $\mathrm{AD}$; therefore, our in silico analysis accurately predicts high risk for SARS-CoV-2 infection in elderly patients with AD (Lim et al., 2020). In addition, our research scheme may be useful for predicting the risk of $\mathrm{AD}$ in patients with SARS-CoV2 infection.

Our findings have implications for the prevention and treatment of SARS-CoV-2 infection in elderly patients with AD. First, both Ace 2 and Nrp1 are preferentially expressed in the brain, and their expression level may determine the sensitivity to SARS-CoV-2 infection (Figure 3C). Interestingly, it was recently suggested that differences in cytokines, such as IL- $1 \beta$ and TNF- $\alpha$, are less pronounced in peripheral blood in SARS-CoV-2 infection (Totura and Baric, 2012; Tincati et al., 2020). Second, in addition to Ace2, Nrp1 expression was also upregulated in patients with severe AD. Although predictive immune biomarkers are suggested for the clinical treatment of COVID-19 (Fouladseresht et al., 2020), our high-throughput analysis-based approach would probably provide an accurate prediction of SARS-CoV-2 risk in elderly AD patients. Notably, Ace2 gene expression gradually increased with the severity of AD symptoms (from incipient to severe stage) (Lim et al., 2020), whereas elevated Nrp1 gene expression was only present in the severe AD patient group (Figures $\mathbf{1 B}, \mathbf{C}$ ). This result indicates that ACE2 may be a more fundamental gene for SARS-CoV-2 infection compared with NRP1.

Recently, the spread of SARS-CoV-2 infection has accelerated worldwide. Efforts on the clinical treatment of SARS-CoV-2 infection are concentrated on the development of vaccines and drugs, including gene therapy (Chugh et al., 2020). To our knowledge, this is the first study examining NRP1 expression in
$\mathrm{AD}$ patients and reporting its higher expression these individuals. Moreover, it reveals the importance of determining SARS-CoV-2 spike protein receptor gene expression. Our gene profiling could potentially be used to predict the risk for SARS-CoV-2 infection in elderly $\mathrm{AD}$ patients.

\section{DATA AVAILABILITY STATEMENT}

The datasets presented in this study can be found in online repositories. The names of the repository/repositories and accession number(s) can be found in the article/Supplementary Material.

\section{ETHICS STATEMENT}

All animal experiments performed in this study were reviewed and approved by the IACUC Committee at Korea Brain Research Institute (IACUC-20-00018). Written informed consent was obtained from the owners for the participation of their animals in this study. Written informed consent was obtained from the individual(s) for the publication of any potentially identifiable images or data included in this article.

\section{AUTHOR CONTRIBUTIONS}

J-YJ and K-HL designed the research. K-HL, SY, S-HK, and J-YJ wrote the manuscript, performed the research, discussed the results, and commented on the manuscript. All authors contributed to the article and approved the submitted version.

\section{FUNDING}

This work was supported by Korea Brain Research Institute (KBRI) basic research program through KBRI funded by the Ministry of Science and ICT (21-BR-02-09, 21-BR-02-21), and Basic Science Research Program through the National Research Foundation of Korea (NRF) funded by the Ministry of Education (2019R1F1A1059595).

\section{ACKNOWLEDGMENTS}

We would like to thank Sun-Woo Lee for assistance of bioinformatics analysis. $5 \times \mathrm{FAD}$ (9-month) mice were kindly provided by Sungkun Chun at Jeonbuk National University Medicine School. A graphic figure was made with biorender.com. We would also like to thank Editage for English language editing.

\section{SUPPLEMENTARY MATERIAL}

The Supplementary Material for this article can be found online at: https://www.frontiersin.org/articles/10.3389/fgene.2021. 741175/full\#supplementary-material 


\section{REFERENCES}

Abdullah, A., Akhand, S. S., Paez, J. S. P., Brown, W., Pan, L., Libring, S., et al. (2020). Epigenetic targeting of neuropilin-1 prevents bypass signaling in drugresistant breast cancer. Oncogene 40, 322-333. doi: 10.1038/s41388-020-0 1530-6

Bianchetti, A., Rozzini, R., Guerini, F., Boffelli, S., Ranieri, P., Minelli, G., et al. (2020). Clinical presentation of COVID19 in dementia patients. J. Nutr. Health Aging 24, 560-562. doi: 10.1007/s12603-020-1389-1

Cantuti-Castelvetri, L., Ojha, R., Pedro, L. D., Djannatian, M., Franz, J., Kuivanen, S., et al. (2020). Neuropilin-1 facilitates SARS-CoV-2 cell entry and infectivity. Science 370, 856-860. doi: 10.1126/science.abd2985

Carstensen, L. L., Shavit, Y. Z., and Barnes, J. T. (2020). Age advantages in emotional experience persist even under threat from the COVID-19 pandemic. Psychol. Sci. 31, 1374-1385. doi: 10.1177/0956797620967261

Chugh, H., Awasthi, A., Agarwal, Y., Gaur, R. K., Dhawan, G., and Chandra, R. (2020). A comprehensive review on potential therapeutics interventions for COVID-19. Eur. J. Pharmacol. 890, 173741. doi: 10.1016/j.ejphar.2020.173741

Daly, J. L., Simonetti, B., Klein, K., Chen, K. E., Williamson, M. K., Anton-Plagaro, C., et al. (2020). Neuropilin-1 is a host factor for SARS-CoV-2 infection. Science 370, 861-865. doi: 10.1126/science.abd3072

Davies, J., Randeva, H. S., Chatha, K., Hall, M., Spandidos, D. A., Karteris, E., et al. (2020). Neuropilin-1 as a new potential SARS-CoV-2 infection mediator implicated in the neurologic features and central nervous system involvement of COVID-19. Mol. Med. Rep. 22, 4221-4226. doi: 10.3892/mmr.2020.11510

Ding, Q., Shults, N. V., Harris, B. T., and Suzuki, Y. J. (2020). Angiotensinconverting enzyme 2 (ACE2) is upregulated in Alzheimer's disease brain. bioRxiv [Preprint]. doi: 10.1101/2020.10.08.331157

Fouladseresht, H., Doroudchi, M., Rokhtabnak, N., Abdolrahimzadehfard, H., Roudgari, A., Sabetian, G., et al. (2020). Predictive monitoring and therapeutic immune biomarkers in the management of clinical complications of COVID19. Cytokine Growth Factor Rev. 58, 32-48. doi: 10.1016/j.cytogfr.2020.10. 002

Fu, L., Wang, B., Yuan, T., Chen, X., Ao, Y., Fitzpatrick, T., et al. (2020). Clinical characteristics of coronavirus disease 2019 (COVID-19) in China: a systematic review and meta-analysis. J. Infect. 80, 656-665. doi: 10.1016/j.jinf.2020.03.041

Hu, B., Guo, H., Zhou, P., and Shi, Z.-L. (2020). Characteristics of SARS-CoV-2 and COVID-19. Nat. Rev. Microbiol. 19, 141-154. doi: 10.1038/s41579-020-004597

Kumanogoh, A., and Kikutani, H. (2013). Immunological functions of the neuropilins and plexins as receptors for semaphorins. Nat. Rev. Immunol. 13, 802-814. doi: $10.1038 /$ nri3545

Lim, K. H., and Joo, J. Y. (2020). Predictive potential of circulating Ube2h mRNA as an E2 ubiquitin-conjugating enzyme for diagnosis or treatment of Alzheimer's disease. Int. J. Mol. Sci. 21:3398. doi: 10.3390/ijms21093398

Lim, K. H., Yang, S., Kim, S. H., and Joo, J. Y. (2020). Elevation of ACE2 as a SARS-CoV-2 entry receptor gene expression in Alzheimer's disease. J. Infect. 81, e33-e34. doi: 10.1016/j.jinf.2020.06.072

Liu, K., Chen, Y., Lin, R., and Han, K. (2020). Clinical features of COVID-19 in elderly patients: a comparison with young and middle-aged patients. J. Infect. 80, e14-e18. doi: 10.1016/j.jinf.2020.03.005

Liu, P.-P., Xie, Y., Meng, X.-Y., and Kang, J.-S. (2019). History and progress of hypotheses and clinical trials for Alzheimer's disease. Signal Trans. Targeted Ther. 4:29. doi: 10.1038/s41392-019-0063-8
Masters, C. L., Bateman, R., Blennow, K., Rowe, C. C., Sperling, R. A., and Cummings, J. L. (2015). Alzheimer's disease. Nat. Rev. Dis. Primers 1:15056. doi: $10.1038 /$ nrdp. 2015.56

Mayi, B. S., Leibowitz, J. A., Woods, A. T., Ammon, K. A., Liu, A. E., and Raja, A. (2021). The role of Neuropilin-1 in COVID-19. PLoS Pathog. 17:e1009153. doi: 10.1371/journal.ppat.1009153

Rahman, M. A., Islam, K., Rahman, S., and Alamin, M. (2020). Neurobiochemical cross-talk between COVID-19 and Alzheimer's disease. Mol. Neurobiol. 58, 1017-1023. doi: 10.1007/s12035-020-02177-w

Singh, D. D., Han, I., Choi, E.-H., and Yadav, D. K. (2020). Recent advances in pathophysiology, drug development and future perspectives of SARS-CoV-2. Front. Cell Dev. Biol. 8:580202. doi: 10.3389/fcell.2020.580202

Song, E., Zhang, C., Israelow, B., Lu-Culligan, A., Prado, A. V., Skriabine, S., et al. (2020). Neuroinvasion of SARS-CoV-2 in human and mouse brain. bioRxiv [Preprint]. doi: 10.1084/jem.20202135

Teesalu, T., Sugahara, K. N., Kotamraju, V. R., and Ruoslahti, E. (2009). C-end rule peptides mediate neuropilin-1-dependent cell, vascular, and tissue penetration. Proc. Natl. Acad. Sci. U.S.A. 106, 16157-16162. doi: 10.1073/pnas.0908 201106

Tincati, C., Cannizzo, E. S., Giacomelli, M., Badolato, R., D’arminio Monforte, A., and Marchetti, G. (2020). Heightened circulating interferon-inducible chemokines, and activated pro-cytolytic Th1-cell phenotype features Covid19 aggravation in the second week of illness. Front. Immunol. 11:580987. doi: 10.3389/fimmu.2020.580987

Totura, A. L., and Baric, R. S. (2012). SARS coronavirus pathogenesis: host innate immune responses and viral antagonism of interferon. Curr. Opin. Virol. 2, 264-275. doi: 10.1016/j.coviro.2012.04.004

Verkhratsky, A., Li, Q., Melino, S., Melino, G., and Shi, Y. (2020). Can COVID-19 pandemic boost the epidemic of neurodegenerative diseases? Biol. Direct 15:28. doi: 10.1186/s13062-020-00282-3

Wu, J. T., Leung, K., Bushman, M., Kishore, N., Niehus, R., De Salazar, P. M., et al. (2020). Estimating clinical severity of COVID-19 from the transmission dynamics in Wuhan. China. Nat. Med. 26, 506-510. doi: 10.1038/s41591-0200822-7

Yin, S., Tong, X., Huang, A., Shen, H., Li, Y., Liu, Y., et al. (2020). Longitudinal anti-SARS-CoV-2 antibody profile and neutralization activity of a COVID-19 patient. J. Infect. 81, e31-e32. doi: 10.1016/j.jinf.2020.06.076

Conflict of Interest: The authors declare that the research was conducted in the absence of any commercial or financial relationships that could be construed as a potential conflict of interest.

Publisher's Note: All claims expressed in this article are solely those of the authors and do not necessarily represent those of their affiliated organizations, or those of the publisher, the editors and the reviewers. Any product that may be evaluated in this article, or claim that may be made by its manufacturer, is not guaranteed or endorsed by the publisher.

Copyright (c) 2021 Lim, Yang, Kim and Joo. This is an open-access article distributed under the terms of the Creative Commons Attribution License (CC BY). The use, distribution or reproduction in other forums is permitted, provided the original author(s) and the copyright owner(s) are credited and that the original publication in this journal is cited, in accordance with accepted academic practice. No use, distribution or reproduction is permitted which does not comply with these terms. 